\title{
Invasive Klebsiella syndrome with coexisting fungal endophthalmitis
}

\author{
Jayanthi Sugantheran, Embong Zunaina, Wan Mariny Md Kasim, Norlaila Talib \\ Sugantheran J, Zunaina E, Md Kasim WM, et al. Invasive Klebsiella syndrome with coexisting fungal endophthalmitis. Malays Fam Physician. \\ 2021;16(2);94-97. https://doi.org/10.51866/cr1100
}

Keywords:

Endophthalmitis, Klebsiella

syndrome, fungal ball,

sphenoid mycetoma

\section{Authors:}

\section{Jayanthi Sugantheran \\ (Corresponding author) \\ Department of Ophthalmology and \\ Visual Science, School of Medical \\ Sciences, Health Campus, Universiti Sains Malaysia, Kelantan, Malaysia Email: jayanthi.dec@gmail.com}

\section{Zunaina Embong}

Department of Ophthalmology and Visual Science, School of Medical Sciences, Health Campus, Universiti Sains Malaysia, Kelantan, Malaysia

\section{Wan Mariny Md Kasim}

Department of Ophthalmology

Hospital Serdang, Jalan Puchong

Kajang, Malaysia

\section{Norlaila Talib}

Department of Ophthalmology, Hospital Serdang, Jalan Puchong Kajang, Malaysia

\begin{abstract}
Endogenous endophthalmitis accounts for approximately $5-10 \%$ of all endophthalmitis cases. We report a case of a middle-aged gentleman with underlying uncontrolled diabetes mellitus who presented with fever and generalised body weakness for one week. He was diagnosed with invasive Klebsiella syndrome based on blood culture with presence of bilateral pleural effusion, liver abscess, renal impairment and sphenoidal sinusitis. The patient developed sudden bilateral painless reduced vision on day two of admission. Ocular examination revealed bilateral severe anterior chamber reaction and severe vitritis that obscured the view of the fundus. Ocular B-scan ultrasonography showed multiple loculations in the posterior segment in both eyes. There was soft tissue density with calcification in the left sphenoid sinus on computed tomography of the orbit. He was treated for bilateral endogenous endophthalmitis with multiple intravitreal antibiotic injections, but showed no improvement. Functional endoscopic sinus surgery was performed and revealed that the left sphenoid sinus was filled with fungal balls. Following drainage of sphenoidal pus, there was resolution of vitritis and fundus examination showed features of underlying fungal infection with a "string of pearls" present along the vascular arcade of both eyes. The patient was diagnosed with bilateral endogenous endophthalmitis secondary to invasive Klebsiella syndrome with coexisting fungal endophthalmitis secondary to sphenoid mycetoma. In addition to repeated intravitreal antibiotic injections, he was also treated with systemic and topical antifungal therapy. At three months post treatment, the infection resolved and his vision improved from counting fingers to 6/36 bilaterally.
\end{abstract}

Key messages: A middle-aged gentleman presented with bilateral endogenous endophthalmitis secondary to invasive Klebsiella syndrome with coexisting fungal endophthalmitis secondary to sphenoid mycetoma. A high index of suspicion is required for early diagnosis of fungal endophthalmitis.

\section{Introduction}

Endophthalmitis is a purulent inflammation of the intraocular fluids (vitreous and aqueous), usually due to infection. Infectious endophthalmitis is one of the most serious complications of ophthalmic surgery. ${ }^{1}$ In this paper, we present a case of bilateral endogenous endophthalmitis secondary to invasive Klebsiella syndrome with coexisting fungal endophthalmitis secondary to sphenoid mycetoma.

\section{Case Presentation}

A 65-year-old male with hypertension and poorly controlled diabetes mellitus (DM) presented to the emergency department with general body weakness for one week associated with fever and vomiting.

Upon examination, the patient was found to be delirious, febrile, tachycardic and tachypnoeic. Baseline blood investigations showed poorly controlled DM with high serum glucose $(24.6 \mathrm{mmol} / \mathrm{l})$ and glycosylated haemoglobin (15.3\%) levels. Total white blood cells were elevated to $12 \times 10^{9}$ (normal: $4-11$ x 109)—predominantly neutrophils (94\%)— with an elevated erythrocyte sedimentation rate of $80 \mathrm{~mm} / \mathrm{hr}$. In addition, there was renal and liver impairment. The patient was admitted to the intensive care unit and treated with 2 $\mathrm{g}$ of intravenous ceftriaxone twice daily for presumed gram-negative sepsis.

The septic workup grew Klebsiella pneumonia from the blood culture, which was sensitive to ceftriaxone and augmentin. Other infective screening results were negative. Given the possibility of masquerade syndrome, tumour markers were taken but all were negative.

The imaging assessment showed bilateral 
pleural effusion on the chest radiograph. Ultrasonography of the abdomen showed the presence of a hypoechoic lesion in the liver, suggesting liver abscess (Figure 1). Computed tomography of the brain and orbit revealed a soft tissue density with calcification in the left sphenoid sinus (Figure 2).

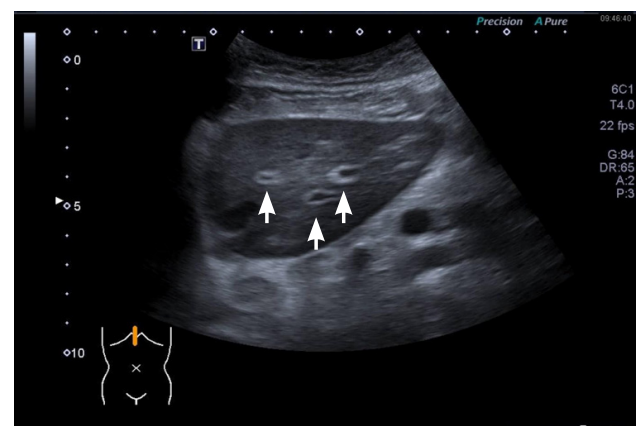

Figure 1: Ultrasonography of abdomen of 65-year-old man showing multiple hypoechoic lesions in the liver at segment 5 (white arrows).

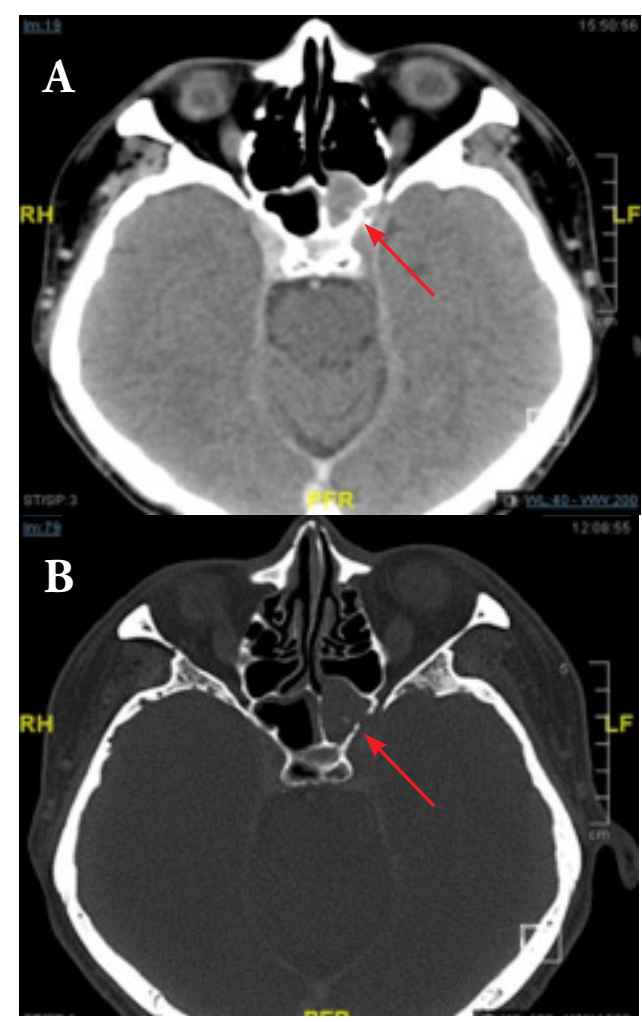

Figure 2: Computed tomography of orbit and paranasal sinus of 65-year-old man showing soft tissue density and calcification within the left posterior sphenoidal sinus with thinning of the sinus wall (red arrow). (A) Soft tissue window, (B) bone window.

Two days following admission, the patient complained of bilateral sudden painless blurring of vision. Upon ocular examination, his visual acuity was counting the toes of one foot with both eyes. The conjunctiva in both eyes were diffusely injected. Both anterior chambers were deep with plasmoid fibrinous aqueous with no hypopyon. Severe vitritis obscured the fundus view. Ocular B-scan ultrasonography showed multiple loculations in the vitreous cavity in both eyes.

The patient's condition was treated as bilateral endogenous endophthalmitis secondary to invasive Klebsiella syndrome. Topical 5\% moxifloxacillin hourly was started for both eyes. Intravitreal tap and intravitreal injection with $2 \mathrm{mg}$ ceftazidime in $0.1 \mathrm{ml}$ and $2 \mathrm{mg}$ vancomycin in $0.1 \mathrm{ml}$ were administered on the same day. Vitreous culture revealed no organism growth and vitreous fluid cytology was acellular.

In view of the absence of ocular improvement at 48 hours post-intravitreal injection of antibiotics and given that the patient was not sufficiently stable for vitrectomy, a second intravitreal antibiotic injection (drug and dosage similar to the first intravitreal injection) was performed, together with a nasal endoscopic procedure by the otolaryngologist team. The nasal endoscopic examination did not, however, show any abnormality. Thus, magnetic resonance imaging of the paranasal sinus was performed, which demonstrated left sphenoidal sinusitis (Figure 3).

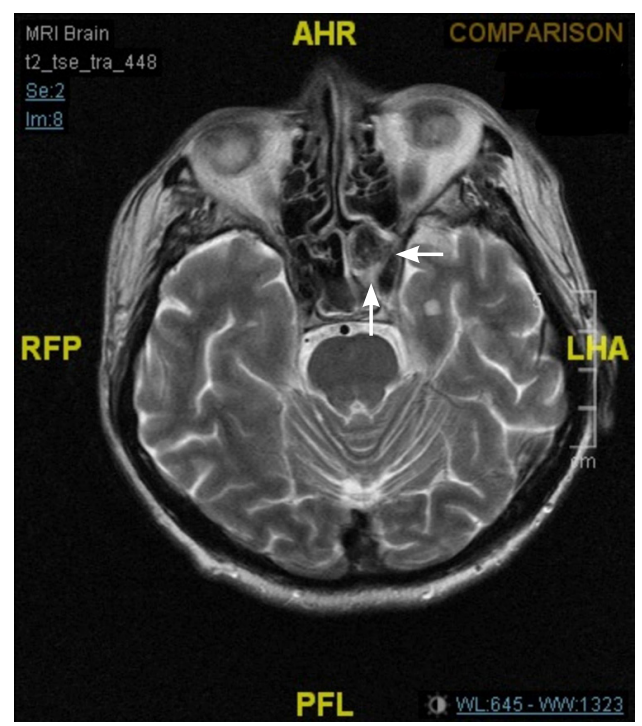

Figure 3: Magnetic resonance imaging of brain and paranasal sinus of 65-year-old man showing left sphenoidal sinusitis (white arrows).

A third dosage of intravitreal antibiotic injection (drug and dosage similar to the first intravitreal injection) was given on the seventh day in view of the slow improvement of the 
ocular infection. On the same day, functional endoscopic sinus surgery was performed, which revealed that the left sphenoid sinus was filled with fungal balls with granulation tissue and oedema of the sphenoid mucosa. The culture of pus from the sphenoid sinus did not grow any organisms.

Anterior chamber inflammation and vitritis resolved following the third intravitreal antibiotic injection and drainage of pus from the sphenoid sinus. Fundus examination showed features of underlying fungal infection, with the presence of a "string of pearls" (whitish opacities) along the vascular arcade of both eyes (Figure 4). Based on this, the patient was diagnosed with bilateral endogenous endophthalmitis secondary to invasive Klebsiella syndrome with coexisting fungal endophthalmitis secondary to sphenoid mycetoma. The patient was treated with $100 \mathrm{mg}$ oral fluconazole twice daily, along with $0.12 \%$ topical amphotericin B and $0.2 \%$ topical fluconazole hourly in both eyes, for over six weeks.

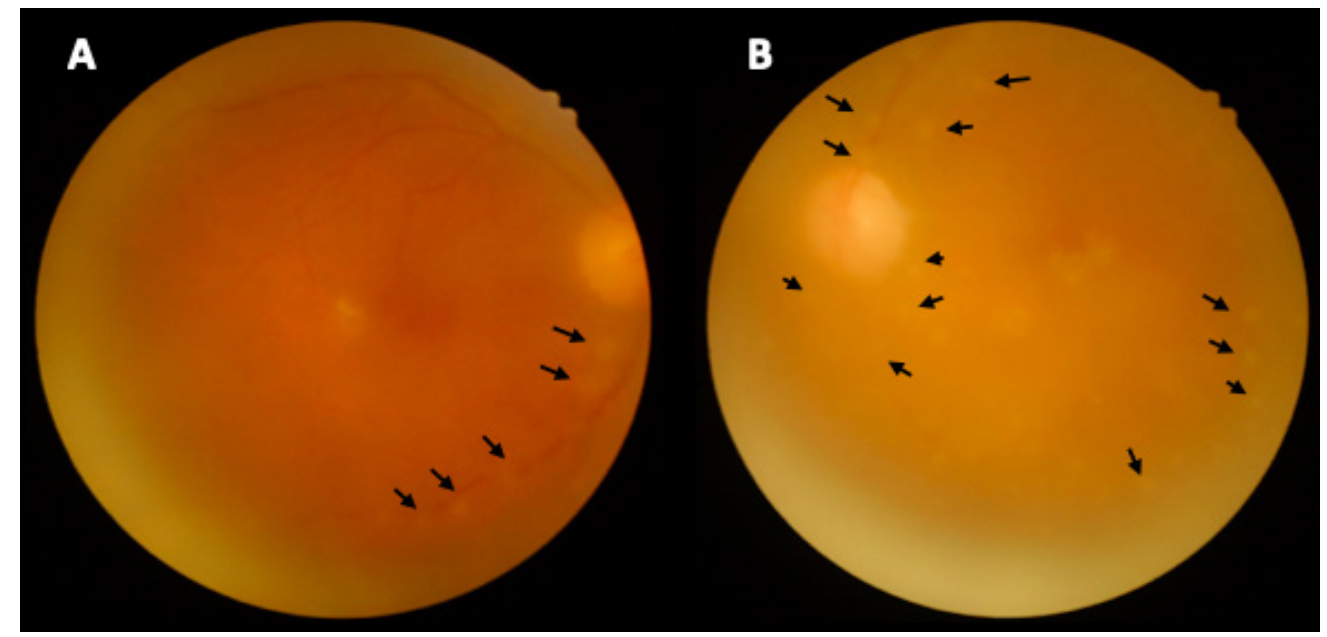

Figure 4: Fundus pictures of 65-year-old man showing presence of vitritis with "string of pearls" along the vascular arcade superiorly and inferiorly in right eye (A) and left eye (B), indicated by black arrows.

Three weeks post-treatment, his vision in both eyes improved to $6 / 36$. The anterior segment revealed mild to moderate cellular reaction. The fundus showed signs that the vitritis was clearing up and the string of whitish opacities began to disappear. Systemically, the blood sugar was controlled with hypoglycaemic agents, the renal and liver function tests showed that their functions had stabilised and pleural effusion was resolved.

\section{Discussion}

Endophthalmitis is a sight-threatening ocular inflammatory response to either infected or injured intraocular tissue. Most cases appear to be due to infectious agents, especially bacteria, although in several reports, culture-negative cases of endophthalmitis predominate. ${ }^{2}$ According to Jackson et al., only $12 \%$ of endogenous endophthalmitis cases are bilateral, as was the case with our patient. ${ }^{2}$

Invasive Klebsiella syndrome is a potentially fatal condition that is also known as septic metastases. Mortality rates range from $25 \%$ to
$37 \% .^{3}$ Klebsiella is a highly virulent intraocular pathogen, with visual outcomes that are worse than counting fingers in $89 \%$ of endogenously infected eyes. ${ }^{4}$ Bilateral endophthalmitis has occurred in one quarter of all reported cases of invasive Klebsiella syndrome. ${ }^{5}$

A fungal ball can be asymptomatic, as in our case, or can simulate chronic rhinosinusitis. A fungal ball is described as a non-invasive accumulation of a dense conglomeration of fungal hyphae in a paranasal sinus. Classically, it involves a single paranasal sinus, similar to our case. $^{6}$

Frequently, blood cultures present a negative result; in fact, in only $23-50 \%$ of cases will cultures aid in the diagnosis. ${ }^{7,8}$ When the fungal ball attacks the sphenoid sinus or the ethmoidal, the choice of treatment is endonasal sphenoid or complete ethmoidectomy, respectively. ${ }^{8}$ Recurrence rate is low when using endoscopic techniques $(6.8 \%)$ and no local or systemic antifungal therapy is needed. ${ }^{8}$ 
In terms of ocular involvement, Candida species are the most common cause of endogenous fungal endophthalmitis, followed by Aspergillus species. ${ }^{9}$ The characteristic findings of Aspergillus endophthalmitis are anterior uveitis with hypopyon and vitreous inflammation, as noted in our patient. Aspergillus has a high propensity for vascular invasion and often causes thrombosis of the infected tissue, but it is quite difficult to culture Aspergillus from infected tissue because it is an extremely fastidious organism. ${ }^{10}$

\section{Conclusion}

The treatment of fungal endophthalmitis poses a serious challenge for ophthalmologists, as the outcome is unfavourable. The main challenge is suspecting fungal aetiology at the time of presentation and accurately diagnosing the patient. Multidisciplinary management is crucial in dealing with multiple organ involvement.

\section{Conflicts of interest}

The authors declare no conflicts of interest. The authors alone are responsible for the content and writing of the paper. Informed consent was obtained from the patient for the publication of the case report. This article does not contain any studies with human or animal subjects.

\section{How does this paper make a difference to general practice?}

- Endophthalmitis is one of the most devastating ocular conditions in ophthalmology.

- Recognising the early signs of endophthalmitis is important so that appropriate treatment can be carried out.

- Failure of early identification and treatment may lead to a fatal course and a guarded visual prognosis.

- Although incidence of bilateral endogenous endophthalmitis is lesser than that of unilateral presentation, clinicians must implement appropriate clinical judgement while also maintaining a low threshold to initiate treatment.

\section{References}

1. Okada AA, Johnson RP, Liles WC, D'Amico DJ, Baker AS. Endogenous bacterial endophthalmitis. Report of a ten-year retrospective study. Ophthalmology. 1994 May; 101(5):832-8.

2. Jackson T, Eykyn S, Graham E, Stanford M. Endogenous bacterial endophthalmitis: A 17-year prospective series and review of 267 reported cases. Surv Ophthalmol, 2003; 48(4):403-423.

3. Forster R, Zachary I, Cottingham A, Norton E. Further observations on the diagnosis, cause, and treatment of endophthalmitis. AJO,1976; 81(1):52-56
4. Leibovici L, Samra Z, Konisberger H. Bacteraemia in adult diabetic patients. Diabetes Care, 1991; 14:89-94.

5. Neetens A, Verschueren C, De SN, Delgadillo R. Bilateral endogenous endophthalmitis. Bull Soc Beige Ophthalmol, 1981; 193:123-30.

6. Nicolai P, Lombardi D, Tomenzoli D, Villaret AB, Piccioni M, Mensi M, Maroldi R. Fungus ball of the paranasal sinuses: Experience in 160 patients treated with endoscopic surgery. Laryngoscope, 2009; 119(11):2275-9.

7. Goodnight J, Dulguerov P, Abemayor E. Calcified mucor fungus ball of the maxillary sinus. Am J Otolaryngol, 1993; 14:209-210.
8. Ferguson BJ. Fungus balls of the paranasal sinuses. Otolaryngol Clin North Am, 2000; 33(2):389-98.

9. Lamaris GA, Esmaeli B, Chamilos G. Fungal endophthalmitis in a tertiary care cancer centre: A review of 23 cases. Eur J Clin Microbiol Infect Dis. 2008; 27:343-347.

10. Rinaldi MG. Invasive aspergillosis. Rev Infect Dis, 1983; 5:1061-77. 